\title{
Evolution of binary stars and its implications for evolutionary population synthesis
}

\author{
Z. Han $^{1}$, X. Chen ${ }^{1}$, F. Zhang ${ }^{1}$ \& Ph. Podsiadlowski ${ }^{2}$ \\ ${ }^{1}$ National Astronomical Observatories/Yunnan Observatory, the Chinese Academy of Sciences, \\ Kunming, 650011, China (email: zhanwenhan@ynao.ac.cn) \\ ${ }^{2}$ University of Oxford, Department of Astrophysics, Keble Road, Oxford, OX1 3RH, UK
}

\begin{abstract}
Most stars are members of binaries, and the evolution of a star in a close binary system differs from that of an ioslated star due to the proximity of its companion star. The components in a binary system interact in many ways and binary evolution leads to the formation of many peculiar stars, including blue stragglers and hot subdwarfs. We will discuss binary evolution and the formation of blue stragglers and hot subdwarfs, and show that those hot objects are important in the study of evolutionary population synthesis (EPS), and conclude that binary interactions should be included in the study of EPS. Indeed, binary interactions make a stellar population younger (hotter), and the far-ultraviolet (UV) excess in elliptical galaxies is shown to be most likely resulted from binary interactions. This has major implications for understanding the evolution of the far-UV excess and elliptical galaxies in general. In particular, it implies that the far-UV excess is not a sign of age, as had been postulated prviously and predicts that it should not be strongly dependent on the metallicity of the population, but exists universally from dwarf ellipticals to giant ellipticals.
\end{abstract}

Keywords. stars: binaries: close - stars: blue stragglers - stars: subdwarfs - galaxies: elliptical and lenticular, $\mathrm{cD}$ - ultraviolet: galaxies

\section{Introduction}

Evolutionary population synthesis (EPS) has experienced a rapid progress since the early 90's and provides the most robust approach in studying stellar populations of galaxies. In most of the current EPS models, binary evolution has been ignored. However, most stars are members of binaries, and binary evolution leads to the formation of many peculiar objects, such as blue stagglers and hot subdwarfs, which are hot, long-lived and still very luminous. Those objects contribute very much to the spectral energy distribution (SED) at short wavelength for an old stellar population, and make the population look hotter and younger. Such a "cosmetic" effect has been successfully included by EPS models of Zhang et al. (2004), Han, Podsiadlowski \& Lynas-Gray (2007), Chen \& Han (2009). Some puzzles in EPS have been solved with the inclusiong of binaries and binary evoltuion is an important ingredient in EPS and also a subject of this symposium.

\section{Binary evolution}

Most stars are in binaries and binaries evolve differently from single stars. Binary evolution is much more complicated and produces many interesting objects, such as cataclysmic variables, type Ia supernovae, X-ray binaries, symbiotic stars, blue stragglers, hot subdwarfs, etc. Binary evolution leads to rejuvenation of stars and the formation of hotter objects in an otherwise old cold population.

There are numerous evolutionary channels for a binary system, depending on its initial conditions. However, the main evolutionary processes can be described as below. A binary 
system (of low/intermediate mass) has two components: the primary (the initially more massive one) and the secondary. As the binary evolves, the primary expands and may fill its Roche lobe, and Roche lobe overflow (RLOF) begins and mass is transferred from primary to secondary. If RLOF is dynamically stable, the mass transfer removes the primary's envelope and its hot core is exposed, and in some cases the core can be ignited (i.e. long-lived). The secondary grows in mass via accretion and this rejuvanates the secondary. Stable RLOF leads to the formation of a wide binary system. If the mass transfer is dynamically unstable, the mass transfer leads to the formation of a common envelope (CE). The CE engulfs the core of the primary and the secondary, and does not co-rotate with the embedded binary. The friction between the $\mathrm{CE}$ and the embedded binary makes the orbit decay, and a large amount of orbital energy released is deposited into the $\mathrm{CE}$. If the $\mathrm{CE}$ can be ejected, a close binary forms with the primary's core being exposed, otherwise the $\mathrm{CE}$ evolution leads to the formation of a fast rotating merger, which is a more massive and hotter star.

As shown above, a binary may produce hotter objects in the following three ways. 1) The envelope of a component is removed via stabel RLOF or CE ejection and leaves a naked (sometime burning) hot core. 2) A star grows in mass via accretion due to stable RLOF and gets rejuvenated. 3) Coalescence of a binary system produces a more massive and hotter star.

\section{Blue stragglers and the contribution to SED}

Blue straggers (BSs) are stars that have remained on the main sequence for a time exceeding that expected, for their masses, from standard stellar evolution theory. BSs are blue and luminous, and may contribute significant excess spectral energy in the blue and ultraviolet of SED of a stellar population. Those objects are relevant to primordial binaries. Binary coalescence from a contact binary is a major channel for single BSs and it is accepted that the contact binaries are mainly from case A mass transfer, which is defined as the primary being on the main sequence at the onset of mass transfer. Another channel to produce BSs from primordial binaries is mass transfer, where the secondary accretes some material and grows in mass. The primary can be on the the main sequence (case A) at the onset of mass transfer, or after the main sequence but before central He burning (case B), or during or after central He burning (case C).

Fig. 1 shows an integrated rest-frame intrinsic SEDs at different ages for a simple stellar population (including binaries) with a mass of $10^{11} M_{\odot}$ at a distance of $10 \mathrm{Mpc}$. The solid lines are for the results without binary interactions considered, and the dotted ones are the contribution of BSs from primordial binary evolution, i.e mass transfer and coalescence of two components (Chen \& Han 2008a, 2008b, 2009).

\section{Hot subdwarfs and the UV-upturn phenomenon of elliptical galaxies}

Hot subdwarfs (including subdwarf B stars, subdwarf O stars and subdwarf OB stars), known as extreme horizontal branch (EHB) stars in globular clusters, are generally considered to be helium-core-burning stars with extremely thin hydrogen envelopes $(<$ $\left.0.02 M_{\odot}\right)$. The leading theory for their formation is the so called binary model proposed by Han et al. (2002, 2003, 2008), which successfully explained the main observational characteristics of field hot subdwarfs and EHB stars in globular clusters. It is now well established that the vast majority of (and quite possibly all) Galactic hot subdwarfs and EHB stars in globular clusters are the results of binary interactions, where a star loses all 


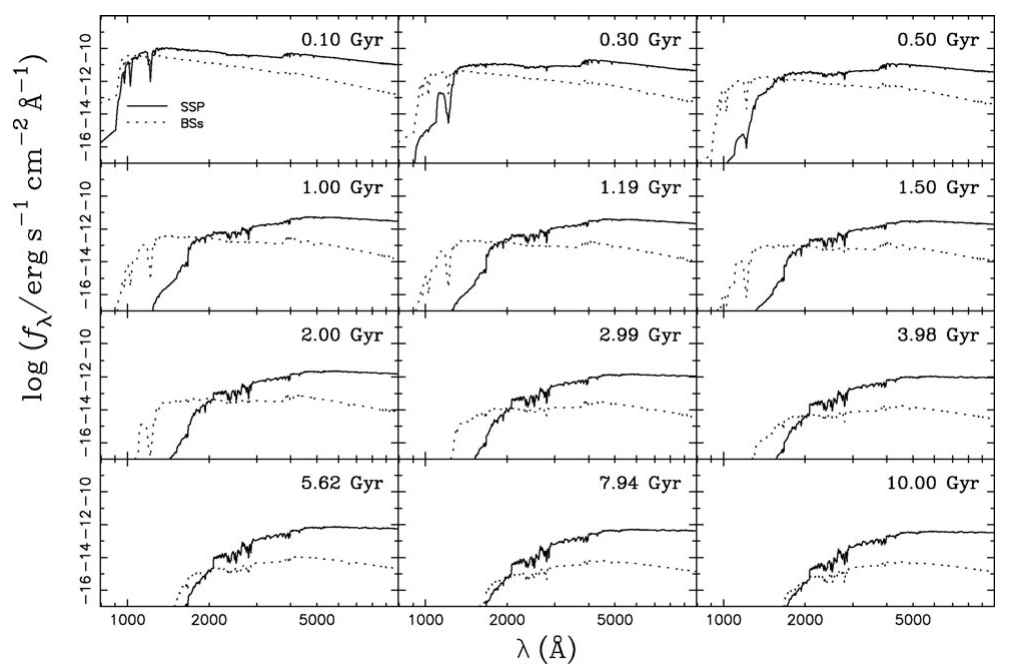

Figure 1. The evolution of SED for a SSP without binary interactions considered (solid line) and the contribution of BSs (dotted line)

of its envelope near the tip of the red-giant branch by mass transfer to a companion star or ejection in a common-envelope phase, or where two helium white dwarfs merge with a combined mass larger than $\sim 0.35 M_{\odot}$ (see Han et al. 2002, 2003, 2008 for references and details). In all of these cases, the remnant star ignites helium and becomes a hot subdwarf. The key feature of these binary channels is that they provide the missing physical mechanism for ejecting the envelope and producing a hot subdwarf. As we will show below, the binary hot subdwarf model provides a natural explanation for the UV-upurn phenomenon of elliptical galaxies.

The UV-upturn phenomenon is a long-standing problem in the study of elliptical galaxies, where there exists a far-ultraviolet (UV) excess in their spectra. It is now clear that UV-upturn is caused by hot subdwarfs. Two scenarios, referred to as the high- and the low-metallicity scenario, had been advanced (Yi, Demarque \& Kim 1997; Lee 1994) before the binary model was invented. In those scenarios, a star loses all of its envelope via stellar wind near the tip of the red giant branch and the naked core gets ignited and becomes a hot subdwarf. These scenarios are ad hoc (see Han, Podsiadlowski \& Lynas-Gray 2007 for details) and require a large age for the hot subdwarfs and therefore predict that the UV excess declines rapidly with redshift. This is not consistent with recent observations, e.g. with the Hubble Space Telescope (HST) (Brown et al. 2003).

The binary model naturally expained the formation of hot subdwarfs, and it would be a priori to apply the binary model of hot subdwarfs to the UV-upturn problem (Han, Podsiadlowski \& Lynas-Gray 2007). Figure 2 shows our simulated evolution of the SED of a galaxy in which all the stars formed at the same time. At early times, the far-UV flux is entirely caused by the contribution from young stars. Hot subdwarfs from the various binary evolution channels become important after about $1.1 \mathrm{Gyr}$, and soon start to dominate completely. After a few Gyr the far-UV SED no longer changes appreciably relative to the visual flux. One immediate implication of this is that the model predicts that the magnitude of the UV excess $(1550-V)$, defined as the relative ratio of the flux in the $V$ band to the far-UV flux, should not evolve significantly with look-back time or redshift. Indeed, this is exactly what seems to have been found in recent observations (Brown et al. 2003, Atlee, Assef \& Kochanek 2009). 


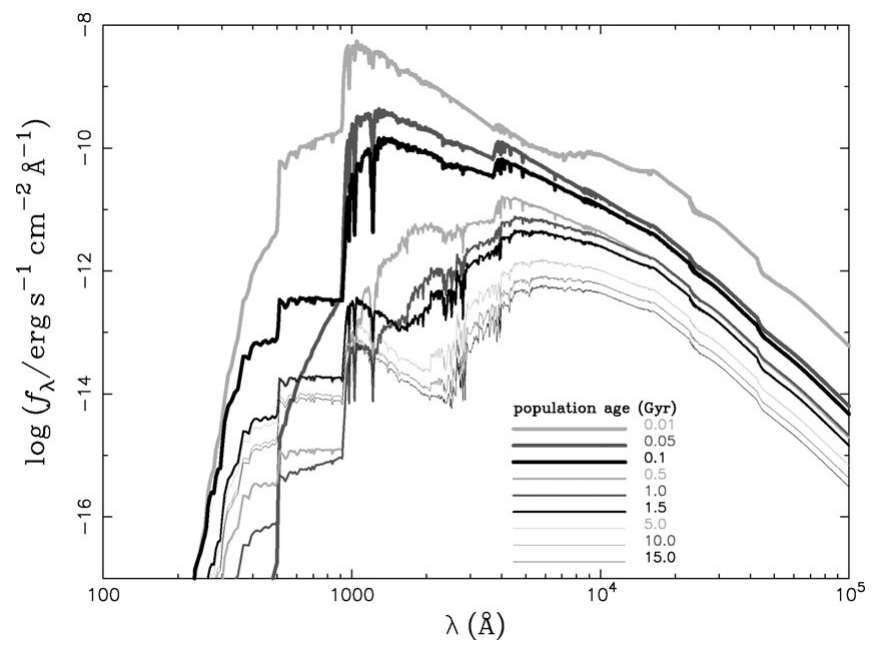

Figure 2. The evolution of the rest-frame intrinsic SED for a simulated galaxy in which all stars formed at the same time, i.e. a simple stellar population (SSP). The stellar population (including binaries) has a mass of $10^{11} M_{\odot}$ and the galaxy is assumed to be at a distance of $10 \mathrm{Mpc}$.

Despite its simplicity, our model can successfully reproduce most of the properties of elliptical galaxies with a UV excess: e.g., the range of observed UV excesses, both in $(1550-V)$ and $(2000-V)$, and their evolution with redshift. The model shows that the UV excess is not a good age indicator, as has been argued previously, and that all old galaxies should show a UV excess at some level. Moreover, we expect that the model is not very sensitive to the metallicity of the population since metallicity does not play a significant role in the envelope ejection process.

Using the binary UV-upturn model, Lisker \& Han (2008) studied the GALEX farnear-UV colours of Virgo Cluster early-type galaxies, and found that, consistent with galaxy formation theories, the opposite behavior in the colours of dwarf ellipticals and giant ellipticals naturally occurs without the requirement of a dichotomy between the stellar populaiton properties of dwarfs and giants, in contrast to previous conclusions.

This work is supported by the Natural Science Foundation of China under grant nos. 10821061, 10973036, 10773026 and 2007CB815406.

\section{References}

Atlee, D. W., Assef, R. J., \& Kochanek, C. S. 2009, ApJ, 694, 1539

Brown, T. M. et al., 2003, ApJ, 584, L69

Chen, X. \& Han, Z. 2008a, MNRAS, 384, 1263

Chen, X. \& Han, Z. 2008b, MNRAS, 387, 1416

Chen, X. \& Han, Z. 2009, MNRAS, 395, 1822

Han, Z., Podsiadlowski, Ph., Maxted, P. F. L., Marsh, T. R., \& Ivanova, N. 2002, MNRAS, 336, 449

Han, Z., Podsiadlowski, Ph., Maxted, P. F. L., \& Marsh, T. R. 2003, MNRAS, 341, 669

Han, Z., Podsiadlowski, Ph., \& Lynas-Gray, A. E. 2007, MNRAS, 380, 1098

Han, Z. 2008, A\&A, 484, L31

Lee, Y. W. 1994, ApJ, 430, L113

Lisker, T. \& Han, Z. 2008, ApJ, 680, 1042

Yi, S.K., Demarque, P., \& Kim, Y. C. 1997, ApJ, 482, 677

Zhang, F., Han, Z., Li, L., \& Hurley, J. R. 2004, A\&A, 415, 117 